ADDENDUM

https://doi.org/10.1038/s41586-018-0112-4

\title{
Addendum: Copper-catalysed enantioselective stereodivergent synthesis of amino alcohols
}

Shi-Liang Shi, Zackary L. Wong \& Stephen L. Buchwald

Addendum to: Nature https://doi.org/10.1038/nature17191, published online 28 March 2016.

In the Supplementary Information of this Letter, we described the use of dimethoxymethylsilane (DMMS), which is used in excess in the reported reaction. This compound is categorized by several vendors as an $\mathrm{H} 318$, category 1 (causing serious eye damage) and by other vendors as an H319, category II (an eye irritant). We have now found a more practical and safer modified method, which requires a small change in the workup procedure. Rather than evaporation of volatiles before workup, the excess DMMS is quenched with ammonium fluoride. This method has been experimentally verified and does not affect the results or conclusions of the original Letter. The Supplementary Information of this Addendum is the modified Supplementary Information, containing the revised workup procedure (pages S7, S23, S29 and S30) but otherwise unchanged.

Supplementary Information is available in the online version of this Addendum. 